\title{
IFN- $\gamma$ increases susceptibility to influenza A infection through suppression of group II innate lymphoid cells
}

\author{
D Califano ${ }^{1,3}$, Y Furuya ${ }^{1,3}$, S Roberts ${ }^{1}$, D Avram ${ }^{1,4}$, ANJ McKenzie ${ }^{2}$ and DW Metzger ${ }^{1}$
}

Increased levels of interferon- $\gamma$ (IFN- $\gamma$ ) are routinely observed in the respiratory tract following influenza virus infection, yet its potential role remains unclear. We now demonstrate that influenza-induced IFN- $\gamma$ restricts protective innate lymphoid cell group II (ILC2) function in the lung following challenge with the pandemic H1N1 A/CA/04/2009 (CA04) influenza virus. Specifically, IFN- $\gamma$ deficiency resulted in enhanced ILC2 activity, characterized by increased production of interleukin (IL)-5 and amphiregulin, and improved tissue integrity, yet no change in ILC2 numbers, viral load or clearance. We further found that IFN- $\gamma$-deficient mice, as well as wild-type animals treated with neutralizing anti-IFN- $\gamma$ antibody, exhibited decreased susceptibility to lethal infection with H1N1 CA04 influenza virus, and moreover that survival was dependent on the presence of IL-5. The beneficial effects of IFN- $\gamma$ neutralization were not observed in ILC2deficient animals. These data support the novel concept that IFN- $\gamma$ can have a detrimental role in the pathogenesis of influenza through a restriction in ILC2 activity. Thus, regulation of ILC2 activity is a potential target for post-infection therapy of influenza.

\section{INTRODUCTION}

Interferon- $\gamma(\mathrm{IFN}-\gamma)$ is a cytokine that is critical for regulating both the innate and adaptive immune response. ${ }^{1}$ Increased production of IFN- $\gamma$ in the respiratory tract is observed during influenza infection, yet its physiological importance remains unclear. ${ }^{2}$ Most studies have indicated that IFN- $\gamma$ is dispensable for survival following influenza infection. ${ }^{3-5}$ However, some reports suggested that IFN- $\gamma$ deficiency can result in increased viral burden or mortality. ${ }^{6-8}$ These discrepancies are likely to be due to differences in experimental design, i.e., viral strain, gene deficiency versus anti-cytokine monoclonal antibody (mAb), and/or immunization routes. Despite this, the general consensus in the field is that deficiency in IFN- $\gamma$ or its receptor does not significantly have an impact on susceptibility in a mouse model of influenza.

Group II innate lymphoid cells (ILC2s) are essential mediators of mucosal immunity, particularly in the lung. ${ }^{9,10}$ ILC2s respond to epithelial cell-derived cytokines, such as interleukin (IL)-33 and IL-25, with rapid production of IL-5 and IL-13, which in turn leads to eosinophil recruitment, mucus secretion, goblet cell hyperplasia, and airway hyperreactivity. ${ }^{11-13}$ Furthermore, lung ILC2s secrete the epidermal growth factor amphiregulin, which promotes epithelial cell proliferation and tissue regeneration following virus-induced tissue damage. ${ }^{10}$ The biological effects of these ILC2-mediated activities can vary, including enhanced development of allergies and asthma, induction of protective immunity against parasitic worms, and regulation of adipocyte development and metabolic homeostasis. ${ }^{14-16}$ During influenza infection, ILC2s are similarly thought to have diverse roles. ${ }^{9,10}$ Considering that large amounts of IFN- $\gamma$ are produced during influenza and that IFN- $\gamma$ can inhibit IL-33-dependent activation of ILC2s, ${ }^{17-19}$ we have now examined the specific role of IFN- $\gamma$ in ILC2 regulation during influenza infection. Our results demonstrate that lethal challenge with the pandemic H1N1 A/CA/04/2009 influenza (CA04) virus caused IFN- $\gamma$-mediated suppression of

${ }^{1}$ Department of Immunology and Microbial Disease, Albany Medical Center, Albany, New York, USA and ${ }^{2}$ Medical Research Council, Laboratory of Molecular Biology, Cambridge, Cambridgeshire, UK. Correspondence: DW Metzger (metzged@mail.amc.edu)

${ }^{3}$ Theses authors are the co-first authors.

${ }^{4}$ Present address: Division of Pulmonary Medicine, Department of Medicine, College of Medicine, University of Florida, Gainesville, Florida 32610-0225, USA. 
ILC2 function in the lung. IFN- $\gamma$ deficiency resulted in enhanced ILC2 activity, characterized by increased production of IL-5 and amphiregulin. Unexpectedly, IFN- $\gamma$-deficient mice, as well as wild-type (WT) animals treated with neutralizing anti-IFN- $\gamma$ antibody, were protected against lethal CA04 infection and increased resistance was dependent on the presence of ILC2s and IL-5. We therefore reveal an unanticipated role for IFN- $\gamma$ in the pathogenesis of CA04 influenza virus infection, specifically in promoting increased susceptibility through the suppression of ILC2 protective responses. We further provide evidence that induced IL-5 and, possibly, increased eosinophilia support survival following lethal viral challenge. These results indicate that specific regulation of ILC2-mediated immune responses could be a promising therapeutic approach for enhancing recovery following influenza infection.

\section{RESULTS}

\section{IFN- $\gamma$ increases susceptibility to CA04 influenza virus challenge}

The role of IFN- $\gamma$ during primary infection with influenza virus remains unresolved. We therefore investigated the response of $\mathrm{BALB} / \mathrm{c}$ IFN $-\gamma^{-1-}$ mice to lethal challenge with the 2009 pandemic strain A/California/04/2009 (CA04) H1N1 virus. $\mathrm{BALB} / \mathrm{c} \mathrm{IFN}-\gamma^{+/+}$mice produced large amounts of IFN $-\gamma$ in the bronchoalveolar lavage fluid (BALF) starting at Day 3 post infection and continuing through Day 9 (Figure 1a). Similar to previous reports, ${ }^{3,20}$ the absence of IFN- $\gamma$ did not have an impact on viral burden (Figure 1a,b); however, BALB/c IFN- $\gamma^{-1-}$ mice surprisingly exhibited increased resistance to lethal infection (Figure 1c), an observation that has never been reported in the literature. ${ }^{3,20}$ This increased resistance was not restricted to a particular mouse strain, as both $\mathrm{BALB} / \mathrm{c}$ and $\mathrm{C} 57 \mathrm{Bl} / 6 \mathrm{IFN}-\gamma^{-1-}$ mice exhibited the same phenotype (Figure 1c,d). The data show that the presence IFN- $\gamma$ caused decreased survival of CA04 virus-infected mice, but this was not due to altered viral clearance.

\section{IFN- $\gamma^{-I-}$ mice exhibit decreased immunopathology}

IFN $-\gamma$ is a pleiotropic cytokine that regulates both the adaptive and innate immune response. We therefore determined whether the increased resistance of BALB/c IFN- $\gamma^{-1-}$ mice was due to an altered immune response. Analysis of influenza virus-specific antibody titers revealed that the levels of IgM, IgG, or IgA anti-CA04 antibodies were comparable between $\mathrm{BALB} / \mathrm{c} \mathrm{IFN}-\gamma^{+1+}$ and IFN- $\gamma^{-1-}$ mice (Supplementary Figure S1a online). We further measured titers of IgG antibody subclasses IgG1, IgG2a, IgG2b, and IgG3 (Supplementary Figure S1b). Antibody titers of the IgG2a and IgG3 isotypes, which are normally stimulated during Th1-type immune responses ${ }^{21}$ were reduced, whereas titers of the Th2-associated IgG1 isotype were modestly increased in BALB/c IFN- $\gamma^{-1-}$ mice (Supplementary Figure S1c), suggesting a predominately type-2 immune response in $\mathrm{BALB} / \mathrm{c}$ IFN- $\gamma^{-1-}$ mice. This was not surprising, as IFN- $\gamma$ is known to be a key switch factor for
IgG2a and IgG3 production. Consistent with the antibody titers, hemagglutination inhibition assays also demonstrated that there were no significant differences in functional antibody responses in $\mathrm{BALB} / \mathrm{c}$ IFN- $\gamma^{+/+}$and IFN- $\gamma^{-1-}$ mice (Supplementary Figure S1c). We observed a transient increase in $\mathrm{CD}^{+}$and $\mathrm{CD}^{+}$T-cell numbers in $\mathrm{BALB} / \mathrm{c}$ IFN- $\gamma^{-1-}$ mice at day 6 post infection (Supplementary Figure S2a); however, granzyme B levels in the BALF were diminished (Supplementary Figure S2b). In addition, levels of inflammatory cytokines in the BALF were comparable in both BALB/c IFN- $\gamma^{+/+}$and IFN- $\gamma^{-1-}$ mice (Supplementary Figure S2c). Thus, in agreement with the similar viral burdens observed in BALB/c IFN- $\gamma^{+/+}$and IFN- $\gamma^{-1-}$ mice, there were no significant differences in antibody or $\mathrm{T}$ - cell responses that could account for the enhanced survival of $\mathrm{BALB} / \mathrm{c}$ IFN- $\gamma^{-1-}$ mice.

Viral respiratory infection can cause massive destruction of the epithelial lung tissue as a result of viral replication, production of inflammatory cytokines by innate and adaptive immune cells, and apoptosis of infected epithelial cells. Recovery from infection therefore not only requires viral clearance but also the regeneration of the lung tissue, which is crucial for normal respiratory function. Taking into account that both $\mathrm{BALB} / \mathrm{c}$ IFN- $\gamma^{+/+}$and IFN- $\gamma^{-1-}$ mice exhibited similar levels of viral lung titers (Figure 1b) and comparable effector immune responses, we postulated that survival differences might be attributed to influenza-induced lung injury. We initially examined lung histopathology but the differences between WT and IFN $-\gamma^{-1-}$ mice were inconclusive. We therefore used quantitative methods of detecting tissue integrity and lung permeability, as these would be suitable for revealing subtle differences. One measure of respiratory distress is pulmonary edema, a correlate of tissue permeability. Pulmonary edema was markedly increased in BALB/c IFN- $\gamma^{+/+}$mice by day 9 following infection. However, $\mathrm{BALB} / \mathrm{c}$ IFN $-\gamma^{-/}$mice presented with a significant reduction in pulmonary edema following infection compared with BALB/c IFN- $\gamma^{+1+}$ mice (Figure 2a). Similarly, albumin and total protein levels in the $\mathrm{BALF}$ of $\mathrm{BALB} / \mathrm{c}$ IFN- $\gamma^{-/-}$mice were significantly reduced when compared with WT mice (Figure $\mathbf{2 b}, \mathbf{c}$ ), further indicating improved tissue integrity in the absence of IFN- $\gamma$. Of note, before day 9 post infection, signs of tissue permeability were equivalent in both BALB/c IFN- $\gamma^{+/+}$and IFN- $\gamma^{-1-}$ animals, suggesting that IFN- $\gamma$-deficient mice exhibit enhanced lung repair following infection (Figure 2a-c). Nitrite levels in the BALF of BALB/c IFN $-\gamma^{-1-}$ mice were likewise reduced, indicating that $\mathrm{BALB} / \mathrm{c}$ IFN $-\gamma^{-1-}$ mice exhibited decreased inflammation following infection with CA04 virus (Figure 2d). In addition, the levels of IL-10 were increased in the absence of IFN- $\gamma$ (Supplementary Figure S2c). Taken together, these data demonstrate that $\mathrm{BALB} / \mathrm{cIFN}-\gamma^{-1-}$ mice exhibit diminished immunopathology and respiratory damage following influenza infection compared with WT animals. These results suggest that the increased resistance of BALB/c IFN- $\gamma^{-1-}$ mice is due not to enhanced viral clearance, but rather to suppressed immunopathology. 
a

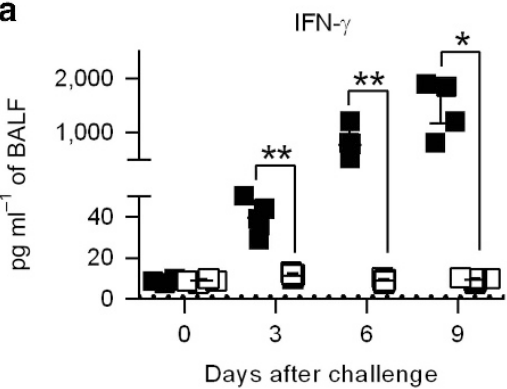

$\mathrm{BALB} / \mathrm{CIFN}-\gamma^{+/+}$(4-5 mice per group) BALB/C IFN- $\gamma^{-1}(4-6$ mice per group)

c

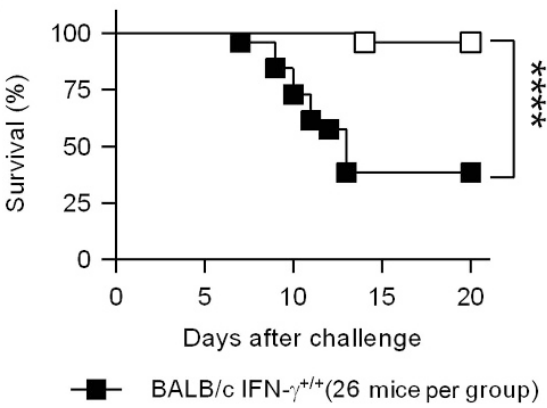

d

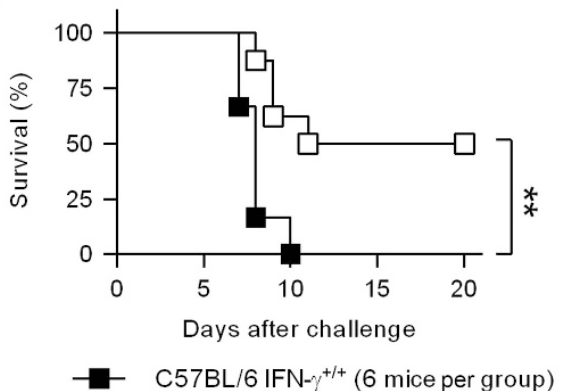

b

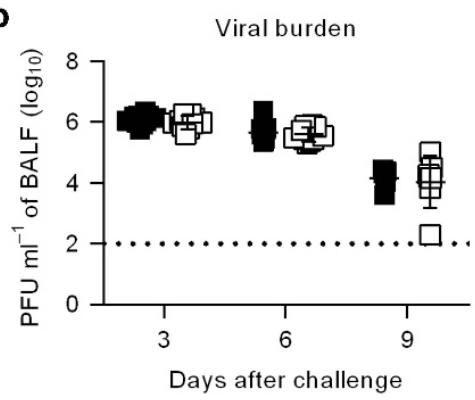

BALB/C IFN- $\gamma^{+/+}$(5-10 mice per group)

$\mathrm{BALB} / \mathrm{C}$ IFN- $\gamma^{-1-}(7-10$ mice per group $)$
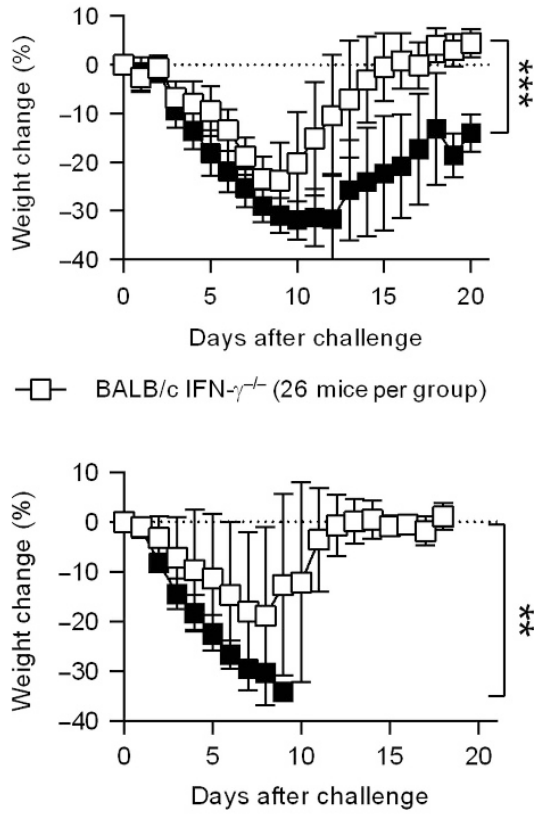

C57BL/6 IFN- $\gamma^{-1-}$ (8 mice per group)

Figure 1 Interferon- $\gamma$ (IFN- $\gamma$ ) promotes mortality during primary A/CA/04/2009 influenza (CA04) infection. (a) Levels of IFN- $\gamma$ in the bronchoalveolar lavage fluid (BALF) of mice infected with 2,000 plaque-forming units (PFUs) of CA04 virus. (b) Pulmonary viral burdens at various time points following CA04 infection. Data shown were pooled from two independent experiments. (c) Survival and weight loss of BALB/c IFN- $\gamma^{+1+}$ and IFN- $\gamma^{-1-}$ mice infected with 2,000 PFUs of the CA04 virus. Data shown were pooled from four independent experiments. (d) Survival and weight loss of C57BL/6 IFN- $\gamma^{+1}$ ${ }^{+}$and IFN- $\gamma^{-1-}$ mice infected with 2,000 PFUs of CA04 virus. (a,b) Statistical analyses were performed by two-way analysis of variance. ${ }^{*} P<0.05$, ${ }^{* *} P<0.01$ and ${ }^{* * * *} P<0.0001$. (c,d) Survival data were analyzed with log-rank Mantel-Cox test and weight loss data were analyzed by Mann-Whitney U-test. ${ }^{* *} P<0.01,{ }^{* \star *} P<0.001$, and ${ }^{* * \star *} P<0.0001$.

\section{ILC2 activity is modulated by IFN- $\gamma$}

The above results indicated that IFN- $\gamma^{-1-}$ mice have both improved tissue integrity and reduced inflammation following influenza infection. However, BALB/c IFN- $\gamma^{-1-}$ mice also exhibited increased airway hyperreactivity (Supplementary Figure S2d). ILC2s have been implicated in both maintaining tissue homeostasis and promoting airway hyperreactivity following influenza infection. ${ }^{9,10}$ We therefore evaluated the ILC2 response after CA04 virus infection. Flow cytometry analysis of intracellular cytokine expression revealed that the lungs and BALF of BALB/c IFN- $\gamma^{-/-}$mice contained significantly greater numbers and percentages of IL- $5^{+}$ILC2s compared with BALB/c IFN- $\gamma^{+/+}$mice (Figure 3a-c and Supplementary Figure S3a). However, the number and percentage of IL- $13^{+}$ILC2s or IL- ${ }^{+}$IL- $13^{+}$ILC2s remained unchanged in the absence of IFN- $\gamma$ (Figure 3a-c and Supplementary Figure S4c-b,e-f). Interestingly, ILC2 production of IL-5 and IL-5 levels in the BALF were not increased in BALB/c IFN- $\gamma^{-1-}$ mice before day 9 post infection (Supplementary Figure S4a,d), which correlates with improved tissue integrity in these mice (Figure 2a-c). The total numbers of ILC2s were equivalent in BALB/C IFN- $\gamma^{+1+}$ and IFN- $\gamma^{-1-}$ mice in both the lung or BALF (Figure 3d, e), suggesting that IFN- $\gamma$ did not have an impact on generation of ILC2s following CA04 virus infection. We further observed major increases in levels of amphiregulin and IL-5, but not IL-13, in the BALF of $\mathrm{BALB} / \mathrm{c} \mathrm{IFN}-\gamma^{-1-}$ mice following CA04 virus infection (Figure $\mathbf{3 f}-\mathbf{h})$. Increased IL-5 production in BALB/c IFN- $\gamma^{-1-}$ mice 
a

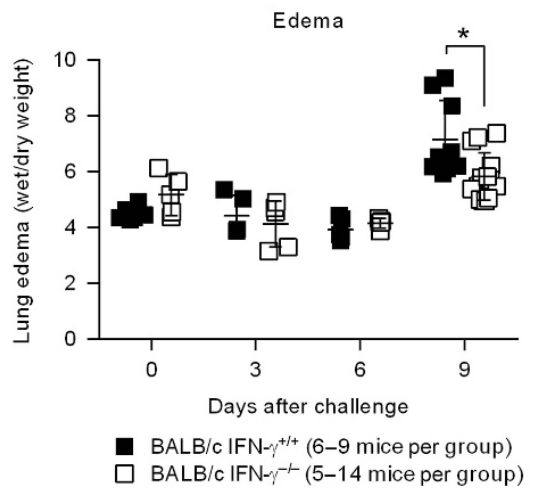

C

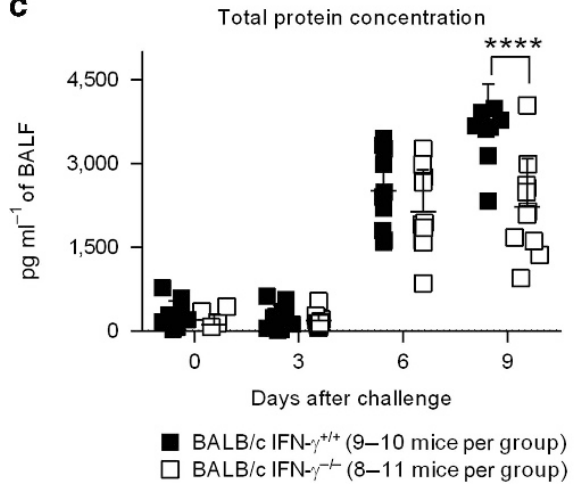

b

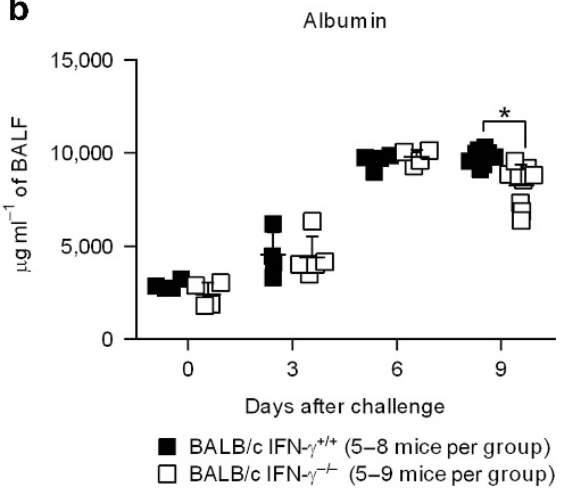

d

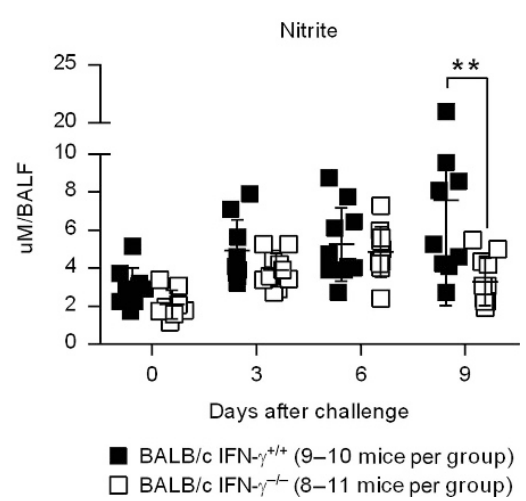

Figure 2 Interferon- $\gamma($ IFN- $\gamma$ ) deficiency reduces immunopathology associated with A/CA/04/2009 influenza (CA04) infection. (a) Pulmonary edema of mice infected with 2,000 plaque-forming units (PFUs) of CA04 virus as determined by tissue wet/dry weight ratio. (b-d) Levels of albumin (b), total protein (c), and nitrite (d) in the BALF of mice infected with 2,000 PFUs of CA04 virus. Data shown were pooled from two independent experiments. (a-d) Statistical analyses were performed by two-way analysis of variance. ${ }^{\star} P<0.05,{ }^{\star \star} P<0.01$, and ${ }^{\star \star \star \star} P<0.0001$.

consequently contributed to enhanced eosinophil recruitment (Figure 3i and Supplementary Figure S3b). Of note, depletion of $\mathrm{CD} 4^{+} \mathrm{T}$ cells using neutralizing antibodies did not have an impact on the levels of amphiregulin, IL-5, or IL-13 in the BALF (Supplementary Figure S5), indicating that Th2 cells were dispensable for the production of these cytokines. Thus, expression of IFN- $\gamma$ strongly correlated with suppression of ILC2 function, specifically IL-5 and amphiregulin expression, and increased lung permeability during CA04 virus infection.

\section{ILC2s express the IFN- $\gamma$ receptor on their surface}

ILC2s become activated in response to alarmin expression by damaged epithelial cells. We therefore evaluated the epithelial cytokines IL-25 and IL-33, which stimulate ILC2 function. ${ }^{11,13}$ Infection with CA04 virus did not trigger IL-25 responses in either BALB/c IFN- $\gamma^{+/+}$or IFN- $\gamma^{-l-}$ mice (Figure 4a), an observation consistent with the literature. ${ }^{22}$ In contrast, IL-33 was highly upregulated following CA04 virus infection but IFN- $\gamma$ deficiency had no impact on IL-33 production (Figure $4 \mathbf{b}$ ) or the expression of ST2 (IL-33R) on the surface of ILC2s (Figure 4c). These data suggest that IFN- $\gamma$ does not suppress ILC2 function through inhibition of IL-33 production or the expression of the IL-33 receptor. To determine whether IFN- $\gamma$ might regulate ILC2 function directly, we evaluated ILC2s for surface expression of IFN- $\gamma$ R1 by flow cytometry. ILC2s from both BALB/c IFN$\gamma^{+/+}$and IFN- $\gamma^{-I-}$ mice expressed similar levels of IFN $-\gamma \mathrm{R} 1$
(Figure 4d). These results, together with previous studies reporting the impact of IFN- $\gamma$ on ILC2 activity, ${ }^{17,19}$ suggest that IFN- $\gamma$ produced during influenza infection modulates ILC2 functions by directly binding to receptors found on the surface of ILC2s.

Diverse groups of ILC2s, such as inflammatory ILC2s, which express IL-17rb and possess the potential to transdifferentiate into ILC3-like cells, have been described. ${ }^{23}$ We therefore evaluated ILCs in lungs for production of ILC1 and ILC3 cytokines. Consistent with the lack of IL-25 production (Figure 4a), we did not observe an increase in inflammatory ILC2s in the lung (data not shown). We further demonstrated comparable numbers of IL- $22^{+} \mathrm{ILCs}$, IL- $22^{+} \mathrm{IL}-17^{+} \mathrm{ILCs}$, and $\mathrm{IL}-17^{+} \mathrm{ILCs}$ in the lungs and BALF of IFN- $\gamma^{+1+}$ and IFN- $\gamma^{-1-}$ mice (Supplementary Figure S6a,b). Similar results were found when we evaluated cytokine levels in the BALF (Supplementary Figure S6c,d). These results indicate that no detectable differences in inflammatory ILC2s or ILC3-like cells were found in $\mathrm{BALB} / \mathrm{c}$ IFN- $\gamma^{+/+}$and IFN- $\gamma^{-1-}$ mice during CA04 virus infection.

\section{Anti-IFN- $\gamma$ treatment promotes resistance to CA04 infection in WT mice}

To further examine the role of IFN- $\gamma$ as a suppressor of ILC2 activity, we treated BALB/c IFN- $\gamma^{+/+}$mice with neutralizing anti-IFN- $\gamma$ antibodies following CA04 infection (Figure 5a,b). 
a

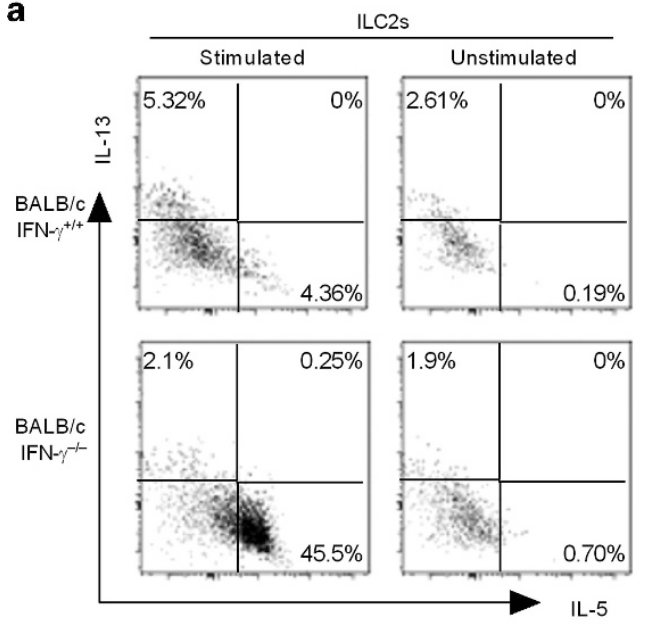

b

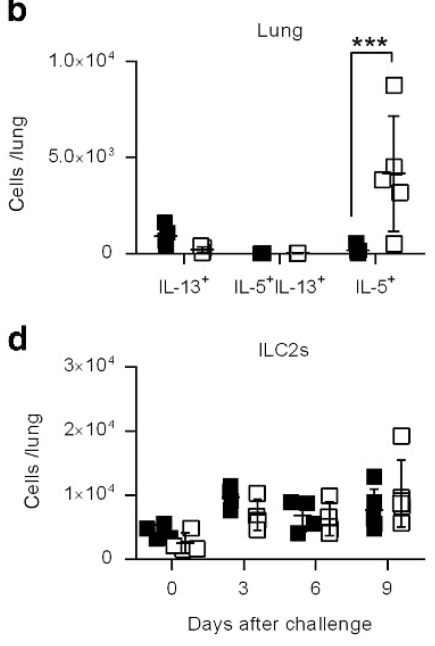

c

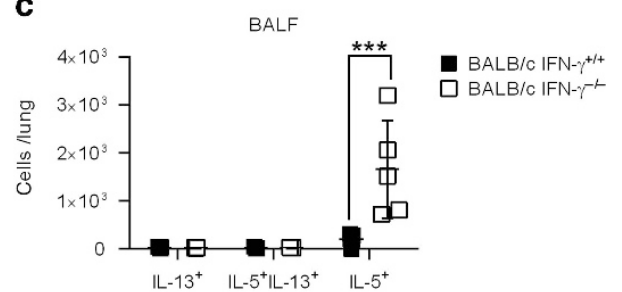

e

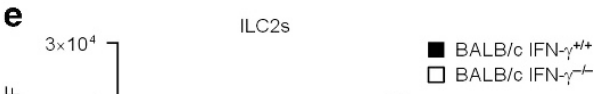

\section{BALB/C IFN $-\gamma^{-1}$}
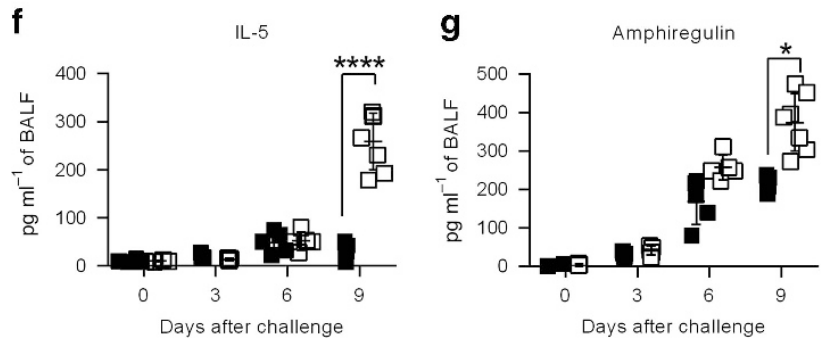
h IL-13
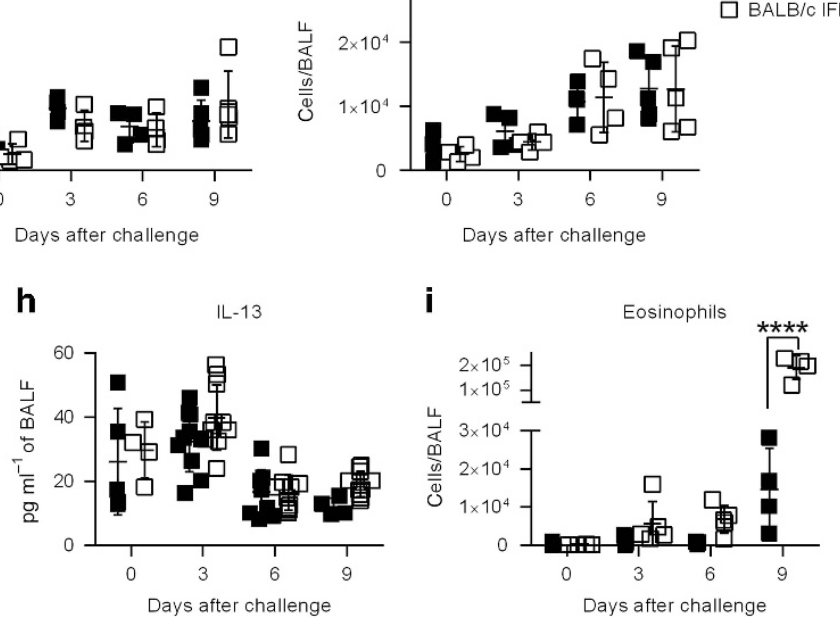

- BALB/C IFN- $\gamma^{+1+} \square$ BALB/C IFN- $\gamma^{-1}$

Figure 3 Interferon- $\gamma$ (IFN- $\gamma$ ) suppresses innate lymphoid cell group II (ILC2) function during A/CA/04/2009 influenza (CA04) infection. (a) Representative dot plots showing percentages of ILC2s $\left(\mathrm{CD} 90^{+} \mathrm{Lin}^{-} \mathrm{CD} 127^{+} \mathrm{KLRG} 1^{+} \mathrm{ST} 2^{+}\right)$that were positive for interleukin (IL)-13 or IL-5. Cells were collected 9 days after CA04 virus infection and restimulated ex vivo with phorbol 12-myristate 13-acetate $\left(50 \mathrm{ng} \mathrm{ml}^{-1}\right)$ and ionomycin $\left(500 \mathrm{ng} \mathrm{ml}{ }^{-1}\right)$ in the presence of Brefeldin $\mathrm{A}\left(10 \mu \mathrm{g} \mathrm{ml}^{-1}\right)$. Unstimulated cells were used as a negative control. $(\mathbf{b}, \mathbf{c})$ Total number of cytokine-secreting ILC2s in the lungs (b) and bronchoalveolar lavage fluid (BALF) (c) 9 days after CA04 infection. (d,e) Total number of ILC2s in the lung (d) and BALF (e). (f-h) Levels of IL-5 (f), Amphiregulin (g), and IL-13 (h) in the BALF of mice infected with 2,000 plaque-forming units (PFUs) of CA04 virus. Data shown were pooled two

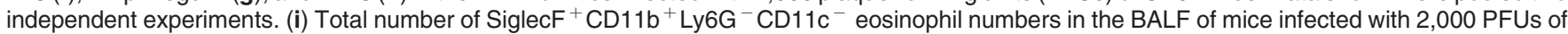
CA04 virus. Data shown were pooled two independent experiments. (b-i) Statistical analyses were performed by two-way analysis of variance. ${ }^{*} P<0.05$, ${ }^{* * *} P<0.001$, and ${ }^{* * \star *} P<0.0001$.

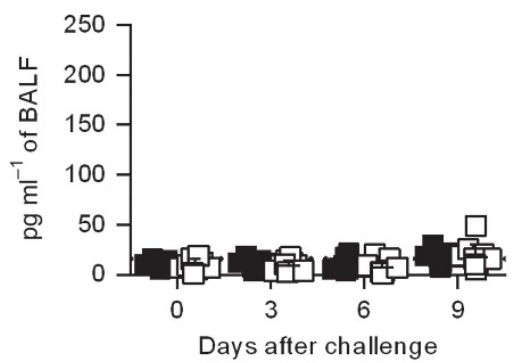

BALB/C IFN- $\gamma^{+/+}$ b

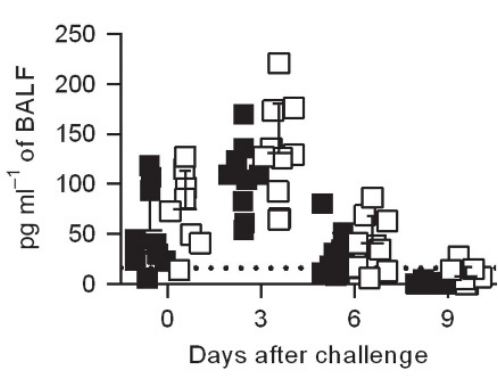

BALB/c IFN- $\gamma^{-1}$ c

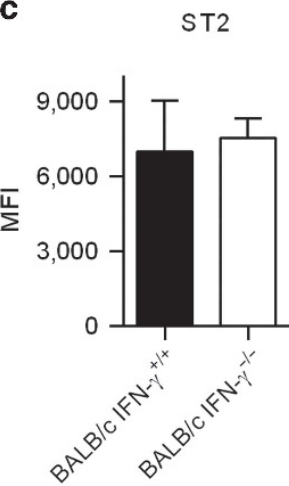

d ILC2s

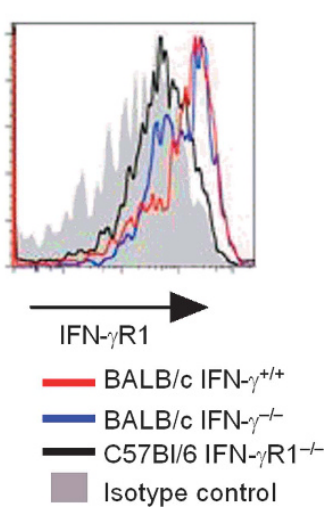

Figure 4 Pulmonary responses and receptor expression on innate lymphoid cell group II (ILC2s) following A/CA/04/2009 influenza (CA04) virus infection. (a,b) Pulmonary interleukin (IL)-25 and IL-33 responses in CA04-infected mice. Data shown were pooled from two independent experiments. (c) Expression of ST2 (IL-33 receptor) on ILC2s (CD90 ${ }^{+} \mathrm{Lin}^{-} \mathrm{CD} 127^{+} \mathrm{KLRG} 1^{+}$). Median fluorescent intensities of five mice per group; representative results from one out of three experiments are shown. (d) A representative histogram of surface expression of interferon- $\gamma$ receptor 1 (IFN- $\gamma$ R1) on ILC2s $\left(\mathrm{CD}_{0}{ }^{+} \mathrm{Lin}^{-} \mathrm{CD}_{127}{ }^{+} \mathrm{KLRG}_{1}{ }^{+} \mathrm{ST} 2^{+}\right)$collected from the lungs of uninfected BALB/c IFN- $\gamma^{+/+}$, BALB/c IFN- $\gamma^{-1-}$, and C57BL/6 IFN- $\gamma \mathrm{R} 1{ }^{-1-}$ mice. $(\mathbf{a}, \mathbf{b})$ Statistical analyses were performed by two-way analysis of variance. (c) Data were analyzed by Mann-Whitney $U$-test. 
a
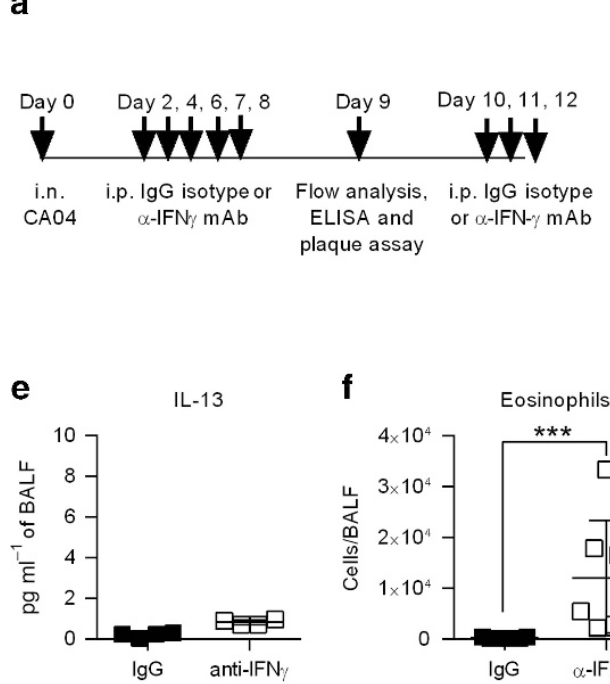

$\mathbf{f}$
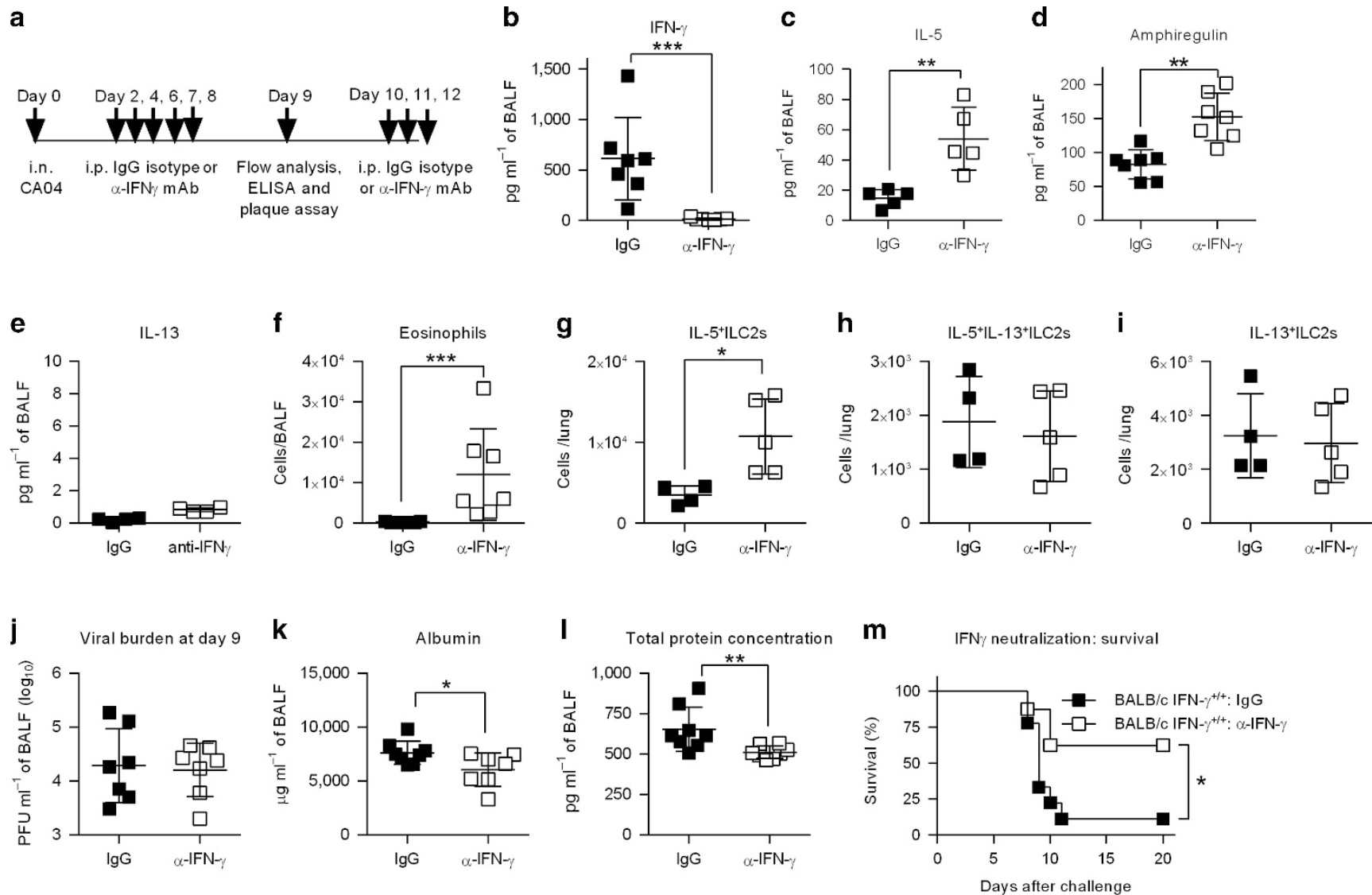

Figure $5 \alpha$-Interferon- $\gamma(\alpha-I F N-\gamma)$ treatment as a therapeutic strategy in BALB/C IFN- $\gamma^{+/+}$mice. (a) Schematic timeline showing $\mathrm{A} / \mathrm{CA} / 04 / 2009$ influenza (CA04) infection, treatment with $\alpha$-IFN- $\gamma$ monoclonal antibody (mAb) or IgG isotype control, and bronchoalveolar lavage fluid (BALF) collection for immunological analyses. (b-f) Quantities of IFN- $\gamma(\mathbf{b})$, interleukin (IL)-5 (c), amphiregulin (d), IL-13 (e), and eosinophils (f) in the BALF of indicated mice 9 days after CA04 virus infection. Data shown were pooled two independent experiments. (g-i) Total numbers IL-5 $5^{+}$ILC2s (g), IL-5 ${ }^{+}$IL-13 ${ }^{+}$ILC2s (h), and IL-13 ${ }^{+}$ILC2s (i) in the lungs of indicated mice 9 days after CA04 infection. (k-m) Viral burden (j), albumin (k), and total protein (I) in the BALF of indicated mice 9 days after CA04 infection. Data shown were pooled two independent experiments. (m) Survival of CA04-infected BALB/c IFN- $\gamma^{+/+}$mice treated with $\alpha-\mathrm{IFN}-\gamma \mathrm{mAb}$ or IgG isotype control. Data shown were pooled two independent experiments. (b-I) Data were analyzed by Mann-Whitney U-test. ${ }^{*} P<0.05,{ }^{* *} P<0.01$, and ${ }^{* * *} P<0.0001$. (m) Survival data were analyzed by log-rank Mantel-Cox test. ${ }^{*} P<0.05$.
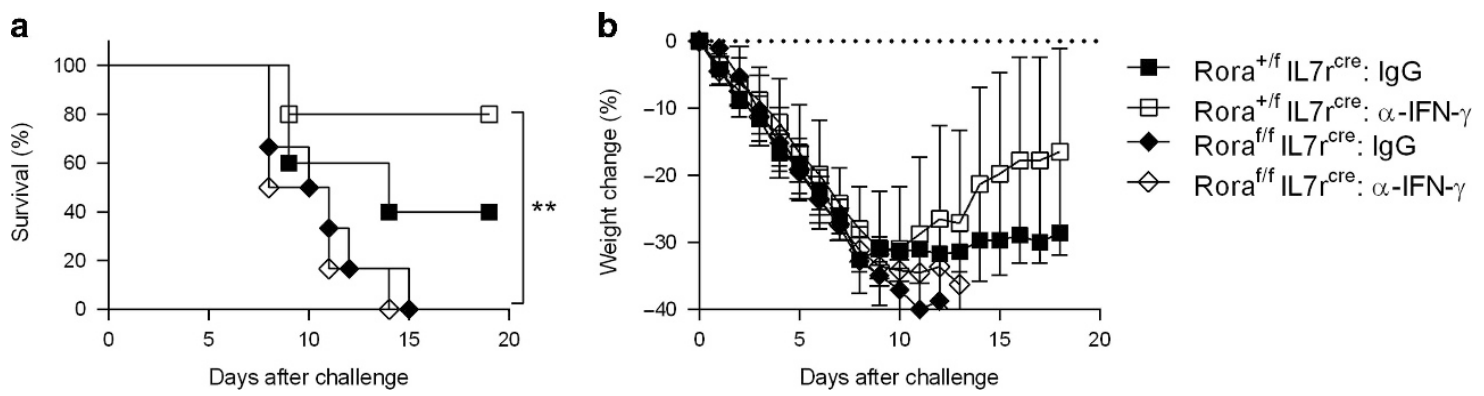

Figure 6 Innate lymphoid cell group II (ILC2s) promote survival following A/CA/04/2009 influenza (CA04) virus infection in the absence of interferon- $\gamma$ (IFN- $\gamma$ ). (a,b) C57BL/6 Rora ${ }^{\mathrm{f} / \mathrm{f}} / / 7 \mathrm{P}^{\mathrm{Cre}}$ and Rora ${ }^{+/ \mathrm{f}} / / 7 \mathrm{P}^{\mathrm{Cre}}$ mice were infected with 500 plaque-forming units (PFUs) of CA04 virus followed by intraperitoneal (i.p.) treatment with $\alpha-I F N-\gamma$ monoclonal antibody (mAb) or IgG isotype control. Survival (a) and weight loss (b) were monitored for 20 days. Survival data were analyzed by log-rank Mantel-Cox test. ${ }^{*} P<0.01$.

IFN- $\gamma$ neutralization caused significant increases in IL-5 and amphiregulin levels in the BALF of infected mice, but IL-13 levels were unaffected (Figure $\mathbf{5} \mathbf{c}-\mathbf{e}$ ). Consistent with elevated production of IL-5, the numbers of eosinophils in the lung were also increased (Figure 5f). Moreover, anti-IFN- $\gamma$-treated mice exhibited an increase in the number of $\mathrm{IL}-5^{+}$ILC2s in the lung (Figure 5g). However, neutralization of IFN- $\gamma$ did not impact the numbers of lung $\mathrm{IL}-13^{+} \mathrm{ILC} 2 \mathrm{~s}$ or $\mathrm{IL}-5^{+}$ IL-13 ${ }^{+}$ILC2s (Figure 5h,i). Similar to BALB/c IFN- $\gamma^{-/-}$ mice, mice treated therapeutically with neutralizing anti-IFN- $\gamma$ antibody did not exhibit changes in viral burden compared to mice treated with IgG isotype control (Figure 5j). Nevertheless, 
neutralization of IFN- $\gamma$ in CA04 virus-infected mice resulted in resistance to infection, as evidenced by diminished levels of BALF albumin and total protein, and increased survival rates, compared to IgG-treated mice (Figure $5 \mathbf{k}-\mathbf{m}$ ). These data, together with results obtained from $\mathrm{BALB} / \mathrm{c}$ IFN- $\gamma^{-/-}$ animals, demonstrate that IFN- $\gamma$ is detrimental during CA04 infection, causing increased morbidity and mortality due to a reduction in tissue integrity.

\section{The beneficial effects of IFN- $\gamma$ neutralization in CA04 virus- infected mice requires ILC2s}

To confirm the protective role of ILC2s in the absence of IFN- $\gamma$ during CA04 virus infection, we evaluated the impact of antiIFN- $\gamma$ on Rora ${ }^{\text {flox/flox }}$ IL $7 r^{\text {cre }}$ mice, which are deficient in ILC2s. ${ }^{24}$ Similar to BALB/c IFN- $\gamma^{+/+}$mice, control litter-

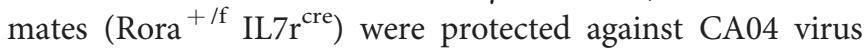
infection following treatment with anti-IFN- $\gamma$ (Figure 6a,b). However, this protection was lost in ILC2-deficient mice (Rora $^{\mathrm{f} / \mathrm{f}}$ IL7r $\mathrm{r}^{\mathrm{cre}}$ ) (Figure 6a,b). Importantly, ILC2-deficient animals showed increased susceptibility to infection compared to WT mice, regardless of the presence of IFN- $\gamma$ (Figure 6a,b). Of note, the survival of IgG isotype-treated control littermates was marginally, but not significantly improved when compared to IgG isotype-treated ILC2-deficient mice (Figure 6a,b), most likely due to the fact that the presence of IFN- $\gamma$ blocks the protective activity of ILC2s. These results indicate that the beneficial effect of IFN- $\gamma$ deficiency is mediated by ILC2s.

\section{IL-5 neutralization restores susceptibility to CA04 virus infection}

To determine whether increased survival from CA04 infection in BALB/c IFN- $\gamma^{-/}$mice was dependent on IL-5, we treated animals post virus infection with either neutralizing anti-IL-5 antibody or IgG isotype control (Figure 7a). BALB/c IFN- $\gamma^{-1-}$ mice treated with anti-IL-5 succumbed to infection around day 9 and had a significantly diminished survival rate when compared with IgG control-treated BALB/c IFN- $\gamma^{-/}$mice (Figure 7b). Neutralization of IL-5 in BALB/c IFN- $\gamma^{-/-}$mice resulted in a major reduction in IL5 ${ }^{+}$ILC2 and eosinophil numbers compared to $\mathrm{BALB} / \mathrm{c}$ IFN- $\gamma^{-/-}$mice treated with IgG isotype control (Figure 7c,d). However, numbers of $\mathrm{IL}_{13}{ }^{+}$ILC2s and $\mathrm{IL} 5^{+} 13^{+}$ILC2s remained consistent in all mouse groups (Supplementary Figure S7a,b online). In addition, numbers of alveolar macrophages, recruited macrophages, and neutrophils were similar between IgG- and anti-IL-5-treated mice (Supplementary Figure S7c-e online). Furthermore, amphiregulin and IL-13 levels, as well as viral burdens, remained unaffected by anti-IL- 5 treatment (Figure $\mathbf{7 f - h}$ ). Inflammation, as measured by the amount of nitrite in the BALF, was slightly increased in the absence of IL-5 (Figure 7i); however, IL-10 levels remained unchanged following anti-IL-5 administration (Supplementary Figure S7f). Indicative of diminished tissue integrity, total protein and albumin levels were also increased in IL-5-neutralized BALB/c IFN- $\gamma^{-/}$mice (Figure $7 \mathbf{j}, \mathbf{k})$. The observed trends are consistent with the involvement of IL-5 in protective lung tissue regeneration. These data support the novel finding that IFN- $\gamma$ production contributes to increased susceptibility to influenza virus infection.

\section{DISCUSSION}

The results presented here establish an unanticipated role for ILC2s in promoting survival during influenza susceptibility and a crucial role for IFN- $\gamma$ as a suppressor of ILC2-mediated protection. Our data further showed that resistance to CA04 virus infection was dependent on the production of IL-5, which appeared to be required to maintain tissue repair postinfection. Gorski et al. ${ }^{22}$ have previously demonstrated that ILC2s produce IL-5 in response to influenza virus infection in WT mice. Interestingly, in Rag $2^{-1-}$ mice, which lack $\mathrm{T}$ and $\mathrm{B}$ cells, and thus exhibit significantly reduced IFN- $\gamma$ production, ${ }^{25}$ levels of IL-5 production are dramatically increased. ${ }^{22}$ Recent data further suggest that IFN- $\gamma$ diminishes ILC2 activity to limit excessive inflammation following helminth infection or asthma exacerbation by fungal infection. ${ }^{18,19}$ However, these reports described a role of IFN- $\gamma$ in terminating ILC2-mediated inflammation and did not characterize/elucidate the regulation of ILC2-mediated functions during an immune response dominated by IFN- $\gamma$, e.g., influenza virus infection. In these previous studies, IFN- $\gamma$ was reported to inhibit production of both IL-5 and IL-13 by ILC2s; ${ }^{18,19}$ however, our results demonstrated that IL-13 production by ILC2s was not enhanced following viral infection. Furthermore, analysis of BALF samples indicated that IL-13 levels were nearly undetectable in either BALB/c IFN- $\gamma^{+/+}$or IFN- $\gamma^{-/-}$mice, suggesting that CA04 virus infection did not elicit a strong IL-13 response. Most evidence presented in the literature suggests that ILC2 expression of IL-5 and IL-13 is regulated concurrently by transcription factors including Gata-3, TCF-1, and Bcl11b. ${ }^{26-29}$ As such, the loss of one or more of these transcription factors results in reduced production of both IL-5 and IL-13. However, the Gfi-1 transcription factor is known to only regulate IL-5 production as evidenced by the fact that genetic ablation of Gfi- 1 causes the specific downregulation of IL- 5 production but not IL-13, ${ }^{30}$ further suggesting that IL-5 and IL-13 are regulated by independent mechanisms.

Monticelli et al. ${ }^{10}$ discovered that ILC2s mediated enhanced lung function and repair following H1N1 (PR8) virus infection through production of amphiregulin. Furthermore, although ILC2s were important for regeneration of lung tissue, they were dispensable for survival following PR8 infection. ${ }^{10}$ Alternatively, our results suggest that in the absence of IFN- $\gamma$, ILC2s are required for improved survival following CA04 virus infection. These findings suggest the concept of a strain-specific contribution of ILC2s to protection against influenza virus infection. Although both PR8 and CA04 are H1N1 influenza viruses, they have distinct origins. ${ }^{31} \mathrm{CA} 04$ virus is derived from the 2009 pandemic strain and is the result of a recombination event involving a Eurasian avian-like swine virus and a triple assortment composed of bird, swine, and human flu viruses. ${ }^{31}$ In addition to its unique origin, the CA04 viral strain possesses a truncated form of the PB1-F2 protein, which is thought to contribute to its reduced pathogenicity. ${ }^{31-33}$ Full-length 
a

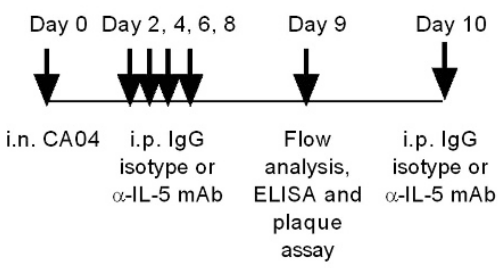

b

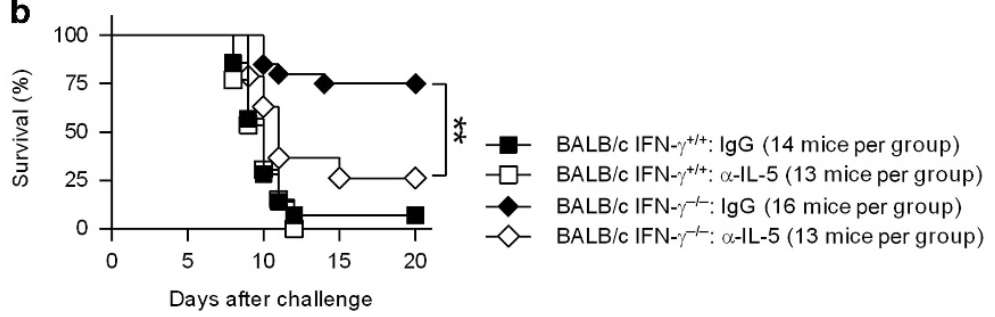

C

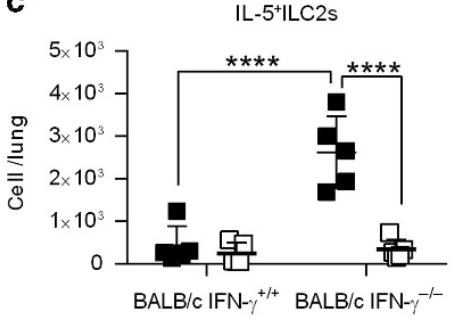

f

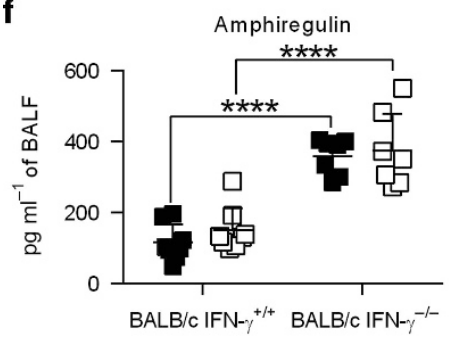

i

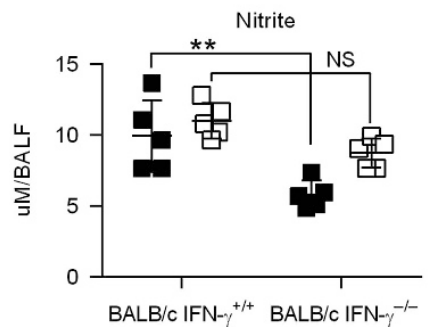

d

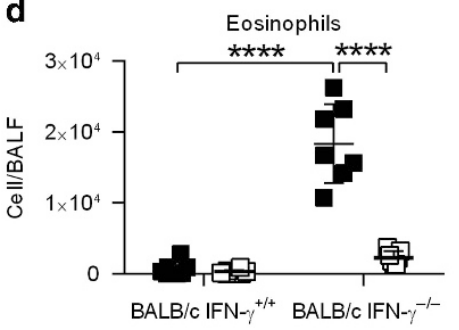

g

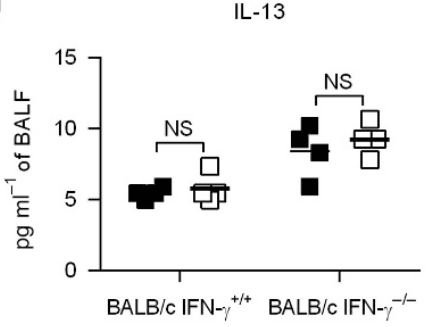

e

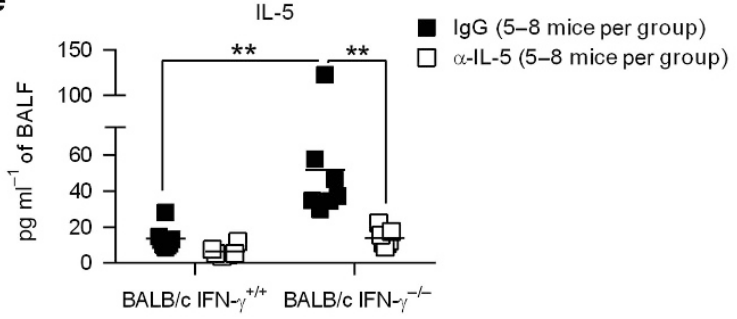

$\mathbf{h}$

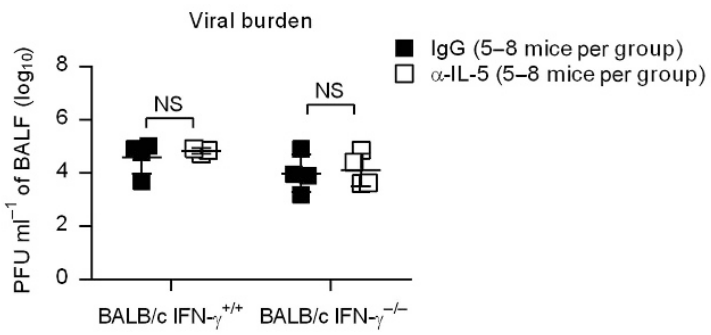

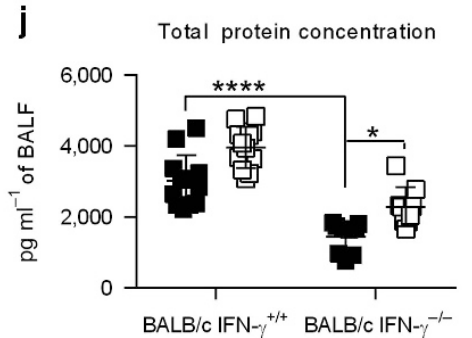

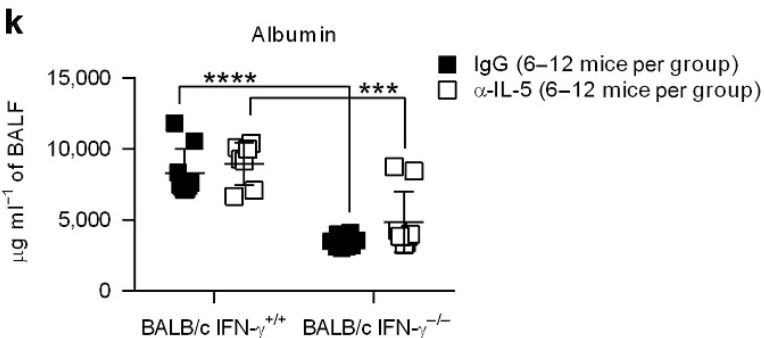

Figure 7 Interleukin (IL)-5 neutralization suppresses the eosinophil response and abrogates resistance of BALB/c IFN- $\gamma^{-1-}$ mice to A/CA/04/2009 influenza (CA04) infection. (a) Schematic timeline showing CA04 infection, treatment with $\alpha$-IL-5 monoclonal antibody (mAb) or lgG isotype control, and bronchoalveolar lavage fluid (BALF) collection for immunological analyses. (b) CA04-infected mice were treated with $\alpha$-IL-5 mAb or IgG isotype control, and monitored for survival. Data shown were pooled from four independent experiments. (c,d) Total numbers of IL-5 $5^{+}$ILC2s (c) and eosinophils (d) in the BALF 9 days after CA04 virus infection of mice treated with $\alpha-I L-5$ mAb or IgG isotype control. (e-g) Levels of IL-5 (e), amphiregulin (f), and IL-13 (g) in the BALF 9 days after CA04 virus infection of mice treated with $\alpha-I L-5$ mAb or IgG isotype control. (h-k) Viral burden (h), nitrite levels (i), total protein (j), and albumin levels $(\mathbf{k})$ in the BALF 9 days after CA04 virus infection of mice treated with $\alpha$-IL-5 mAb or IgG isotype control. (b) Survival data were analyzed with log-rank Mantel-Cox test. ${ }^{* \star} P<0.01$. (c-k) Statistical analyses were performed by two-way analysis of variance. ${ }^{* \star} P<0.01,{ }^{* * *} P<0.001$, and ${ }^{* \star * \star} P<0.0001$. Data pooled from two independent experiments.

PB1-F2, which is expressed in PR8 as well as in isolates derived from the 1918 influenza virus, promotes apoptosis of immune cells, contributes to increased immunopathology, and negatively impacts viral clearance. ${ }^{31-34}$ As a result, the PR8 virus exhibits significantly increased virulence compared with the 2009 pandemic strain, which may overcome ILC2-mediated protection.

IFN $-\gamma^{-/}$mice were protected against lethal challenge with CA04 virus, without substantial differences in viral burden when compared with WT animals. BALB/c IFN- $\gamma^{-/-}$mice also exhibited lower levels of total protein content and albumin in the BALF, indicating decreased epithelial and/or endothelial barrier permeability. This suggests that enhanced survival was most likely to be dependent upon improved tissue integrity and not increased viral clearance. ILC2 production of amphiregulin is known to support lung repair and tissue homeostasis following viral infection, ${ }^{35,10}$ and although we demonstrated that mice lacking IFN- $\gamma$ exhibited elevated levels of amphiregulin in the BALF following viral infection, our data suggested that increased production of IL-5 in the absence of IFN- $\gamma$ was most crucial to improved survival following CA04 virus infection. In support of this, antibody-mediated 
neutralization of IL-5 resulted in increased susceptibility accompanied by a mild reduction in tissue integrity. These findings reveal a possible unexpected role for IL-5 in airway epithelial cell regeneration and tissue maintenance following lung injury, resulting in enhanced survival following influenza infection. However, neutralization of $\mathrm{IL}-5$ in $\mathrm{BALB} / \mathrm{c}$ IFN- $\gamma^{-1-}$ mice did not significantly alter lung permeability or inflammation. As previously described, ${ }^{10}$ the presence of amphiregulin probably contributed to the maintenance of tissue regeneration, but was not sufficient by itself to promote survival. Further studies will be required to elucidate the precise mechanisms of IL-5-mediated protection.

In addition to IL-5, survival may be dependent on the presence of pulmonary eosinophils. Eosinophils promote allergic inflammation and asthma in the lung, but published evidence suggests that they may also enhance anti-viral resistance during respiratory syncytial virus ${ }^{36}$ and parainfluenza virus ${ }^{37}$ infections. Although eosinophils are primarily known for their destructive impact on tissue epithelium, previous studies have shown that increased eosinophilia may actually limit airway hypersensitivity during viral infection. ${ }^{36}$ Moreover, eosinophils have been reported to reduce inflammation and promote tissue homeostasis. ${ }^{38}$ Furthermore, recent evidence suggests that eosinophils have an important role in restricting ILC2 plasticity during Th1 immune responses. ${ }^{39}$ Thus, it is possible that recruitment of eosinophils can contribute to ILC2 stability during influenza infection. Whether eosinophils participate in protection against CA04 virus infection requires further exploration.

Based on our results and the work of others, ${ }^{17-19}$ we postulate that IFN- $\gamma$ acts directly on ILC2s, signaling through the IFN- $\gamma$ receptor to alter their function. Nevertheless, it is also possible that IFN- $\gamma$ blocks ILC2 function through a bystander mechanism, possibly through interference with the IL-33 signaling pathway. As evidenced by our data, IL-33 secretion is stimulated after influenza infection both in the presence and absence of IFN- $\gamma$; therefore, one possibility is that IFN- $\gamma$ limits the influence of IL-33 on ILC2 activity. The IL-33 receptor, a member of the Toll-like/IL-1 receptor super family, is composed of IL-1 receptor accessory protein and ST2L, an isoform of ST2 that contains an extracellular domain, a transmembrane region and a cytoplasmic domain. ${ }^{40-43}$ It has been reported that soluble ST2, a secreted isoform of ST2, which lacks the transmembrane and cytoplasmic regions, sequesters IL-33 to suppress signaling and activation of ILC2s. ${ }^{44}$ Mast cells produce soluble ST2 following FceRI activation ${ }^{45}$ and moreover IFN- $\gamma$ regulates FceRI-dependent mast cell activities during allergic airway inflammation, ${ }^{46}$ indicating a possible role for IFN- $\gamma$ in the expression of soluble ST2 by mast cells. The potential indirect impact of IFN- $\gamma$ on ILC2 activity is an area for further investigation.

In summary, we report the novel finding that IFN- $\gamma$ production contributes to increased susceptibility to influenza virus infection. IFN- $\gamma$ appears to have a major role as a suppressor of ILC2-mediated protection, namely in restricting the production of IL-5. Inhibition of IL-5 with neutralizing antibodies resulted in reduced survival in IFN- $\gamma$-deficient mice, indicating that protection was dependent on the production of IL-5 and possibly increased eosinophilia. These results establish an unanticipated role for ILC2s in promoting resistance against influenza infection. Therefore, specific regulation of ILC2 activity during infection could ultimately prove to be a potent avenue for the development of more effective influenza treatments, as well as enhanced vaccination procedures.

\section{METHODS}

Mouse strains and viruses. $\mathrm{BALB} / \mathrm{c}$ and $\mathrm{C} 57 \mathrm{Bl} / 6 \mathrm{IFN}-\gamma^{+/+}$and IFN- $\gamma^{-1-}$ mice were originally obtained from Jackson Laboratories (Bar Harbor, ME). Rora ${ }^{+ \text {flox }} I l 7 r^{\mathrm{Cre}}$ mice were generated in the lab of Dr. Andrew N.J. McKenzie (MRC Laboratory of Molecular Biology). ${ }^{24}$ All mice were maintained under specific pathogen-free conditions in the Animal Research Facility at Albany Medical College. Mice were anesthetized by intraperitoneal injection with $100 \mu \mathrm{l}$ xylazine $\left(20 \mathrm{mg} \mathrm{ml}^{-1}\right)$ and ketamine $\left(1 \mathrm{mg} \mathrm{ml}^{-1}\right)$ in phosphate-buffered saline. For influenza infections, anesthetized IFN- $\gamma^{+/+}$and IFN $-\gamma^{-l}$ mice were intranasally inoculated with a lethal dose $(2,000$ plaque-forming units) H1N1 A/California/04/2009 (CA04). ILC2deficient animals and their littermate controls $\left(\right.$ Rora $\left.^{+/ \text {flox }} \mathrm{IlTr}^{\mathrm{Cre}}\right)$ were given the median lethal dose of 500 plaque-forming units. Survival and weight loss were monitored daily for 20 days. The virus was propagated in 10-day-old embryonated chicken eggs (Charles River, Wilmington, MA). The stock titer was measured by standard plaque assay. All experimental procedures were approved by the Institutional Animal Care and Use Committee at Albany Medical College (Protocol Number 11-04004).

Pulmonary viral burdens. Influenza viral loads were measured in BALF samples by plaque assay on Madin-Darby canine kidney cells.

Analysis of lung lavage samples. To collect BALF samples, a small incision was made in the trachea and an 18-gauge cannula was inserted. Lungs were lavaged with $1 \mathrm{ml}$ of phosphate-buffered saline and samples were centrifuged to remove cells. Cells were used for flow cytometry analysis. Cell-free supernatants were analyzed for protein levels of IL-2, IL-4, IL-5, IL-10, IL-12, tumor necrosis factor, and IFN- $\gamma$ using Bio-Plex mouse cytokine assays (Bio-Rad, Hercules, CA). TGF $\beta 1$, amphiregulin, IL-25, IL-33, and IFN- $\gamma$ levels were also measured using commercially available kits from BD Biosciences (San Jose, CA), eBioscience (San Diego, CA), and R\&D Systems (Minneapolis, MN). Total protein concentrations in the cell free fractions of BALF were determined using a Pierce BCA protein assay kit (Thermo Scientific, Rockford, IL). Nitrite levels were quantified using a colorimetric Griess reagent kit (Life Technologies, Carlsbad, CA). Levels of albumin in BALF were determined with the BCG albumin assay kit (Sigma-Aldrich, St. Louis, MO).

Measurement of pulmonary edema. Whole mouse lungs were harvested on day 9 post infection. Lung weight (wet) was measured using an AX105 Delta Range analytical balance (Mettler Toledo, Columbus, $\mathrm{OH}$ ) followed by $72 \mathrm{~h}$ incubation at $60^{\circ} \mathrm{C}$. Dried lungs were weighed and edema was expressed as a ratio of lung wet/dry weight. ${ }^{47}$

Hemagglutination inhibition assay. Heat-inactivated BALF samples from CA04 infected mice were serially diluted and incubated with $50 \mu \mathrm{l}$ of CA04 (4 HAU) in a 96-well plate at room temperature for $30 \mathrm{~min}$. Chicken red blood cells (Hemostat Laboratories, Dixon, CA) was added to each well and incubated at room temperature for an additional hour. Hemagglutination inhibition titers were determined as the last reciprocal sample dilutions that fully inhibited influenza virus hemagglutination.

Characterization of lung ILC2s. Lungs were perfused by injecting $10 \mathrm{ml}$ phosphate-buffered saline through the right ventricle, followed 
by digestion of the lung tissue in phosphate-buffered saline buffer containing DNAse I $\left(150 \mu \mathrm{g} \mathrm{ml}^{-1}\right.$; Roche) and collagenase D (1 mg g ml${ }^{-1}$; Roche) for $45 \mathrm{~min}$ at $37^{\circ} \mathrm{C}$. Following digestion, lung tissue was passed through a $40 \mu \mathrm{m}$ filter and blood cells were subsequently removed by incubation in ACK buffer. ILC2s were identified using flow cytometry as cells which expressed CD90.2 (Thy1.2), CD127 (IL-7R), ST2 (IL-33R), and KLRG1, while excluding any cells that expressed lineage-specific markers for $\mathrm{T}$ cells (CD3), B cells (CD45R), myeloid cells (TER-119, CD11b, and Gr-1), and dendritic cells (CD11c).

Flow cytometric analysis. BALF cells or lung single cell suspensions were first incubated with $\alpha$-mouse Fc $\gamma \mathrm{III} / \mathrm{II}$ receptor $(2.4 \mathrm{G} 2 \mathrm{mAb})$ for $15 \mathrm{~min}$. Fc receptor-blocked cells were then stained with mixtures of anti-mouse mAbs. Cells were further stained with Fixable Viability Dye (eFluor 780; eBioscience) to label dead cells. For intracellular cytokine staining, cells were stimulated ex vivo for five hrs with $50 \mathrm{ng} \mathrm{ml}^{-1}$ phorbol 12 -myristate 13 -acetate and $500 \mathrm{ng} \mathrm{ml}^{-1}$ ionomycin at $37^{\circ} \mathrm{C}$, in the presence of $10 \mu \mathrm{g} \mathrm{ml}^{-1}$ Brefeldin A. Cells were stained for surface markers, followed by fixation and permeabilization in $4 \%$ paraformaldehyde and $0.05 \%$ saponin buffer, respectively, followed by intracellular cytokine staining. Stained cells were analyzed on a BD FACSCanto or LSR II (BD Biosciences, San Jose, CA). Data were analyzed using FlowJo software (Tree Star Inc., Ashland, OR).

In vivo cytokine neutralization. For IL-5 neutralization, BALB/c IFN $\gamma^{+1+}$ and IFN $\gamma^{-1-}$ mice were intraperitoneally treated with $150 \mu \mathrm{g}$ of $\alpha$-IL-5 mAb (clone TRFK5; BioXcell, West Lebanon, NH) or IgG isotype control on days $2,4,6,8$, and 10 post infection. The efficacy of eosinophil depletion and IL-5 neutralization in the BALF was confirmed on day 9 post infection by flow cytometry and MILLIPLEX MAP mouse cytokine multiplex assay, respectively. For IFN- $\gamma$ neutralization, mice were intraperitoneally injected with $600 \mu \mathrm{g} \alpha$ IFN- $\gamma \mathrm{mAb}$ (clone XMG1.2; BioXcell) or IgG isotype control on days 2, $4,6,7,8,10,11$, and 12 post infection. The efficiency of IFN- $\gamma$ neutralization in the BALF was determined 9 days after infection by enzyme-linked immunosorbent assay. For CD4 depletion, mice were intraperitoneally injected with $500 \mu \mathrm{g} \alpha$-CD4 mAb (clone GK1.5; BioXcell) or IgG isotype control on days 2, 4, 6, and 8 post infection. The efficiency of CD4 depletion was confirmed by fluorescenceactivated cell sorting analysis.

Airway responsiveness. Airway responsiveness to increasing doses of methacholine was measured on day 10 post infection, as previously described. ${ }^{48}$ Infected mice were anesthetized, tracheostomized, paralyzed with pancuronium bromide, and then connected to a computer-controlled small-animal ventilator (flexiVent; SCIREQ, Montreal, Canada).

Antibodies. Cells were analyzed using the following antibodies: $\alpha$ CD4 (clone GK1.5) (allophycocyanin; BD Pharmingen, San Jose, CA), $\alpha$-CD8 (clone 53-6.7) (phycoerythrin (PE); BD Pharmingen), $\alpha$-CD19 (clone 6D5) (PerCP-Cy5.5; Biolegend, San Diego, CA), $\alpha$-CD11b (clone M1/70) (fluorescein isothiocyanate; eBioscience), $\alpha$-Siglec $\mathrm{F}$ (clone E50-2440) (PE; BD Pharmingen), $\alpha$-Ly6G (clone 1A8) (PE-Cy7; Biolegend), $\alpha$-CD11c (clone HLC) (allophycocyanin; BD Pharmingen), $\alpha$-CD3 $\varepsilon$ (clone 145-2C11) (Biotin; eBioscience), $\alpha$-TER-119 (clone TER-119) (Biotin; eBioscience), $\alpha$-Gr1 (clone RB6-8C5) (Biotin; eBioscience), $\alpha$-CD45R (clone RA3-6B2) (Biotin; eBioscience), $\alpha$-CD11b (clone M1/70) (Biotin; eBioscience), $\alpha$-CD11c (clone N418) (Biotin; eBioscience), $\alpha$-CD90.2 (clone 53-2.1) (PE-Cy7; eBioscience), $\alpha$-CD127 (clone A7R34) (allophycocyanin-eFluor 780; eBioscience), $\alpha$-ST2 (clone DJ8) (fluorescein isothiocyanate; MDBioproducts, Zurich, Switzerland), $\alpha$-IL-5 (clone TRFK5) (PE; eBioscience), $\alpha$-IL-13 (clone eBio13A) (eFluor 660; eBioscience), anti-IL17 (clone ebio17B7) (fluorescein isothiocyanate; eBioscience), anti-IL22 (clone IL22JOP) (allophycocyanin; eBioscience), and Streptavidin (PerCP-Cy5.5; eBioscience).
Statistics. All statistical calculations were performed using GraphPad Prism (La Jolla, CA). Tests between two groups used a two-tailed Mann-Whitney $U$-test. Tests between multiple groups used two-way analysis of variance with Bonferroni's multiple comparisons. Survival curves were evaluated by log-rank Mantel-Cox test and weight loss data were analyzed by using a two-tailed Mann-Whitney U-test.

SUPPLEMENTARY MATERIAL is linked to the online version of the paper at http://www.nature.com/mi

\section{ACKNOWLEDGMENTS}

We thank Dr Katherine C. MacNamara for providing IFN- $\gamma \mathrm{R} 1^{-/-}$mice. We also thank the Department of Immunology and Microbial Disease Immunology Core Laboratory for their technical assistance. This work was supported by NIH grant RO1 Al75312 to D.W.M., NIH grant R01 A1067846 to D.A. and by American Lung Association Biomedical Research Grant RG-341974 to Y.F.

\section{AUTHOR CONTRIBUTIONS}

D.C., Y.F., and S.R. conducted the experiments and analyzed the data. D.A. advised on the experimental procedures. D.C., Y.F., and D.W.M. conceived and designed the experiments. A.N.J.M. provided ILC2-deficient mice. D.C., Y.F., S.R., D.A., and D.W.M. wrote the paper.

\section{DISCLOSURE}

The authors declared no conflict of interest.

c 2018 Society for Mucosal Immunology

\section{REFERENCES}

1. Farrar, M.A. \& Schreiber, R.D. The molecular cell biology of interferongamma and its receptor. Annu. Rev. Immunol. 11, 571-611 (1993).

2. Price, G.E., Gaszewska-Mastarlarz, A. \& Moskophidis, D. The role of alpha/ beta and gamma interferons in development of immunity to influenza A virus in mice. J. Virol. 74, 3996-4003 (2000).

3. Graham, M.B., Dalton, D.K., Giltinan, D., Braciale, V.L., Stewart, T.A. \& Braciale, T.J. Response to influenza infection in mice with a targeted disruption in the interferon gamma gene. J. Exp. Med. 178, 1725-1732 (1993).

4. Nguyen, H.H., van Ginkel, F.W., Vu, H.L., Novak, M.J., McGhee, J.R. \& Mestecky, J. Gamma interferon is not required for mucosal cytotoxic T-lymphocyte responses or heterosubtypic immunity to influenza A virus infection in mice. J. Virol. 74, 5495-5501 (2000).

5. Turner, S.J., Olivas, E., Gutierrez, A., Diaz, G. \& Doherty, P.C. Disregulated influenza A virus-specific CD8 + T cell homeostasis in the absence of IFNgamma signaling. J. Immunol. 178, 7616-7622 (2007).

6. Bot, A., Bot, S. \& Bona, C.A. Protective role of gamma interferon during the recall response to influenza virus. J. Virol. 72, 6637-6645 (1998).

7. Karupiah, G., Chen, J.H., Mahalingam, S., Nathan, C.F. \& MacMicking, J.D. Rapid interferon gamma-dependent clearance of influenza A virus and protection from consolidating pneumonitis in nitric oxide synthase 2-deficient mice. J. Exp. Med. 188, 1541-1546 (1998).

8. Weiss, I.D. et al. IFN-gamma treatment at early stages of influenza virus infection protects mice from death in a NK cell-dependent manner. J. Interferon Cytokine Res. 30, 439-449 (2010).

9. Chang, Y.J. et al. Innate lymphoid cells mediate influenza-induced airway hyper-reactivity independently of adaptive immunity. Nat. Immunol. 12, 631-638 (2011).

10. Monticelli, L.A. et al. Innate Iymphoid cells promote lung-tissue homeostasis after infection with influenza virus. Nat. Immunol. 12, 1045-1054 (2011).

11. Saenz, S.A. et al. IL25 elicits a multipotent progenitor cell population that promotes $\mathrm{T}(\mathrm{H}) 2$ cytokine responses. Nature 464, 1362-1366 (2010).

12. Barlow, J.L. et al. Innate IL-13-producing nuocytes arise during allergic lung inflammation and contribute to airways hyperreactivity. J. Allergy Clin. Immunol. 129: 191-198) e191-e194 (2012).

13. Bartemes, K.R., lijima, K., Kobayashi, T., Kephart, G.M., McKenzie, A.N. \& Kita, H. IL-33-responsive lineage- CD25 + CD44(hi) lymphoid cells 
mediate innate type 2 immunity and allergic inflammation in the lungs. $J$. Immunol. 188, 1503-1513 (2012).

14. Molofsky, A.B. et al. Innate lymphoid type 2 cells sustain visceral adipose tissue eosinophils and alternatively activated macrophages. J. Exp. Med. 210, 535-549 (2013).

15. Halim, T.Y. et al. Group 2 innate lymphoid cells are critical for the initiation of adaptive Thelper 2 cell-mediated allergic lung inflammation. Immunity 40 , 425-435 (2014).

16. Mirchandani, A.S. et al. Type 2 innate lymphoid cells drive CD4 + Th2 cell responses. J. Immunol. 192, 2442-2448 (2014).

17. Molofsky, A.B. et al. Interleukin-33 and interferon-gamma counter-regulate group 2 innate lymphoid cell activation during immune perturbation. Immunity 43, 161-174 (2015).

18. Duerr, C.U. et al. Type linterferon restricts type 2 immunopathology through the regulation of group 2 innate lymphoid cells. Nat. Immunol. 17, 65-75 (2016).

19. Moro, K. et al. Interferon and IL-27 antagonize the function of group 2 innate lymphoid cells and type 2 innate immune responses. Nat. Immunol. 17, 76-86 (2016).

20. Sarawar, S.R., Sangster, M., Coffman, R.L. \& Doherty, P.C. Administration of anti-IFN-gamma antibody to beta 2-microglobulin-deficient mice delays influenza virus clearance but does not switch the response to a Thelper cell 2 phenotype. J. Immunol. 153, 1246-1253 (1994).

21. Snapper, C.M. \& Paul, W.E. Interferon-gamma and B cell stimulatory factor-1 reciprocally regulate Ig isotype production. Science $236,944-947$ (1987).

22. Gorski, S.A., Hahn, Y.S. \& Braciale, T.J. Group 2 innate lymphoid cell production of IL-5 is regulated by NKT cells during influenza virus infection. PLoS Pathog. 9, e1003615 (2013).

23. Huang, Y. et al. IL-25-responsive, lineage-negative KLRG1(hi) cells are multipotential 'inflammatory' type 2 innate lymphoid cells. Nat. Immunol. 16, 161-169 (2015).

24. Oliphant, C.J. et al. MHCIl-mediated dialog between group 2 innate lymphoid cells and $\mathrm{CD} 4(+) \mathrm{T}$ cells potentiates type 2 immunity and promotes parasitic helminth expulsion. Immunity 41, 283-295 (2014).

25. Sun, K. \& Metzger, D.W. Inhibition of pulmonary antibacterial defense by interferon-gamma during recovery from influenza infection. Nat. Med. 14, 558-564 (2008).

26. Hoyler, T. et al. The transcription factor GATA-3 controls cell fate and maintenance of type 2 innate lymphoid cells. Immunity 37, 634-648 (2012).

27. Mjosberg, J. et al. The transcription factor GATA3 is essential for the function of human type 2 innate lymphoid cells. Immunity 37, 649-659 (2012).

28. Mielke, L.A. et al. TCF-1 controls ILC2 and NKp46+RORgammat + innate lymphocyte differentiation and protection in intestinal inflammation. J. Immunol. 191, 4383-4391 (2013).

29. Califano, D. et al. Transcription factor Bcl11b controls identity and function of mature type 2 innate lymphoid cells. Immunity 43, 354-368 (2015).

30. Spooner, C.J. et al. Specification of type 2 innate lymphocytes by the transcriptional determinant Gfi1. Nat. Immunol. 14, 1229-1236 (2013).
31. Neumann, G., Noda, T. \& Kawaoka, Y. Emergence and pandemic potential of swine-origin H1N1 influenza virus. Nature 459, 931-939 (2009).

32. Zamarin, D., Ortigoza, M.B. \& Palese, P. Influenza A virus PB1-F2 protein contributes to viral pathogenesis in mice. J. Virol. 80, 7976-7983 (2006).

33. Pena, L. et al. Restored PB1-F2 in the 2009 pandemic $\mathrm{H} 1 \mathrm{~N} 1$ influenza virus has minimal effects in swine. J. Virol. 86, 5523-5532 (2012).

34. Chen, W. et al. A novel influenza A virus mitochondrial protein that induces cell death. Nat. Med. 7, 1306-1312 (2001).

35. Zaiss, D.M., Yang, L., Shah, P.R., Kobie, J.J., Urban, J.F. \& Mosmann, T.R. Amphiregulin, a TH2 cytokine enhancing resistance to nematodes. Science 314, 1746 (2006).

36. Phipps, S. et al. Eosinophils contribute to innate antiviral immunity and promote clearance of respiratory syncytial virus. Blood 110, 1578-1586 (2007).

37. Adamko, D.J., Yost, B.L., Gleich, G.J., Fryer, A.D. \& Jacoby, D.B. Ovalbumin sensitization changes the inflammatory response to subsequent parainfluenza infection. Eosinophils mediate airway hyperresponsiveness, $\mathrm{m}(2)$ muscarinic receptor dysfunction, and antiviral effects. J. Exp. Med. 190, 1465-1478 (1999).

38. Sugawara, R. et al. Small intestinal eosinophils regulate Th17 cells by producing IL-1 receptor antagonist. J. Exp. Med. 213, 555-567 (2016).

39. Bal, S.M. et al. IL-1 beta, IL-4 and IL-12 control the fate of group 2 innate lymphoid cells in human airway inflammation in the lungs. Nat. Immunol. 17, 636-645 (2016).

40. Tominaga, S. A putative protein of a growth specific cDNA from BALB/c3T3 cells is highly similar to the extracellular portion of mouse interleukin 1 receptor. FEBS Lett. 258, 301-304 (1989).

41. Yanagisawa, K., Takagi, T., Tsukamoto, T., Tetsuka, T. \& Tominaga, S. Presence of a novel primary response gene ST2L, encoding a product highly similar to the interleukin 1 receptor type 1. FEBS Lett. 318, 83-87 (1993).

42. Sims, J.E. \& Dower, S.K. Interleukin-1 receptors. Eur. Cytokine Netw. 5, 539-546 (1994).

43. Greenfeder, S.A., Nunes, P., Kwee, L., Labow, M., Chizzonite, R.A. \& Ju, G. Molecular cloning and characterization of a second subunit of the interleukin 1 receptor complex. J. Biol. Chem. 270, 13757-13765 (1995).

44. Hayakawa, H., Hayakawa, M., Kume, A. \& Tominaga, S. Soluble ST2 blocks interleukin-33 signaling in allergic airway inflammation. J. Biol. Chem. 282, 26369-26380 (2007).

45. Bandara, G., Beaven, M.A., Olivera, A., Gilfillan, A.M. \& Metcalfe, D.D. Activated mast cells synthesize and release soluble ST2-a decoy receptor for IL-33. Eur. J. Immunol. 45, 3034-3044 (2015).

46. Yu, M. etal. Identification of an IFN-gamma/mast cell axis in a mouse model of chronic asthma. J. Clin. Invest. 121, 3133-3143 (2011).

47. Tate, M.D., Pickett, D.L., van Rooijen, N., Brooks, A.G. \& Reading, P.C. Critical role of airway macrophages in modulating disease severity during influenza virus infection of mice. J. Virol. 84, 7569-7580 (2010).

48. Durrant, D.M., Gaffen, S.L., Riesenfeld, E.P., Irvin, C.G. \& Metzger, D.W. Development of allergen-induced airway inflammation in the absence of T-bet regulation is dependent on IL-17. J. Immunol. 183, 5293-5300 (2009). 